Classification

Physics Abstracts

79.60

\title{
Electronic structures of clean and sulphur saturated Pt (100) surfaces
}

\author{
T. T. A. Nguyen and R. C. Cinti \\ Groupe des Transitions de Phases, C.N.R.S., BP 166, 38042 Grenoble Cedex, France
}

(Reçu le 25 janvier 1982, révisé le 2 mars, accepté le 5 mars 1982)

\begin{abstract}
Résumé. - Les mesures de photoémission uv angulaire ont été effectuées sur une surface de $\mathrm{Pt}(100)$ propre puis saturée de $S$ adsorbé, avec des énergies d'excitation comprises entre 10,2 et 40,8 eV. Pour la surface propre, les résultats expérimentaux sont en bon accord avec la structure de bande du Pt calculée récemment par McDonald et al. L'adsorption de $\mathrm{S}$ introduit deux structures électroniques supplémentaires assez similaires à celles généralement observées dans la chimisorption des chalcogènes sur métaux de transition.
\end{abstract}

\begin{abstract}
Angle-resolved uv photoemission experiments have been performed on clean and $\mathbf{S}$ saturated Pt (100) surface, in the photon energy range 10.2-40.8 eV. Empirical energy bands along the $\Gamma \mathrm{X}$ direction of the Brillouin zone are in good agreement with the calculated scheme for Pt given recently by McDonald et al. Adsorption spectra show two extra electronic structures, similar to those generally observed with chalcogen chemisorption on transition metals.
\end{abstract}

Angle-resolved uv photoemission (ARUPS) has become a common technique for determining the electronic structure of crystalline surfaces. Among metals, copper, silver and nickel are the most investigated. A considerable number of existing calculations for both clean and adsorbed surfaces of these metals have permitted fruitful comparisons between theory and experiments and also explorations of the capability of the technique. Similar studies with $\mathrm{Pt}$ are more seldom. Empirical valence band structures have been drawn for Pt (111) by Cinti et al. [1] and by Mills et al. [2], for Pt (100) by Thornton et al. [3]. One of the difficulties encountered in these studies was the determination of the final states participating in the interband transitions. We have found that by using the quasi-free electron conduction band given by Smith [4] our experimental dispersion relation $E(\mathbf{k})$ is in very good agreement with the valence band structure calculated recently for Pt by McDonald et al. [5]. On the other hand, chalcogen adsorption on Pt has been the subject of controversy. By angle-integrated UPS, Collins and Spicer [6] have found no oxygen resonant state on polycrystalline and two vicinal Pt surfaces at $\hbar \omega=10.2$ and $21.2 \mathrm{eV}$. They suggested that the chemisorption bond of oxygen on platinum would be different from that on $3 \mathrm{~d}$ transition metals, $\mathrm{Ni}$ and $\mathrm{Fe}$, while Küppers and Ertl[7] argued the contrary. For sulphur adsorbed on $\mathrm{Pt}(100)$ surface, we have detected two extra electronic structures very similar to those observed previously for oxygen or sulphur adsorption on $\mathrm{Ni}(100)$ [8].

Experiments were carried out in an ultrahigh-vacuum spectrometer described previously [1]. The electron analyser was a CMA the aperture of which was partially obstructed in order to have 
the full acceptance angle of $10^{\circ}$. The collection angle $\theta$ relative to the surface normal was varied by rotating the sample around an axis in the surface plane, arcs $\theta$ projecting onto the [001] azimuthal direction. By using a grazing incidence holographic grating (Jobin-Yvon LHT 30) and four discharge gases $\left(\mathrm{H}_{2}, \mathrm{~A}, \mathrm{Ne}\right.$ and $\left.\mathrm{He}\right)$, twelve resonance lines sufficiently strong for photoemission purpose were obtained in the photon energy range from $10.2 \mathrm{eV}$ to $40.8 \mathrm{eV}$. In situ cleaning of the crystal was performed by heat treatment in oxygen and repetitive cycles of ion-bombardmentannealing [1] until no trace of contamination was detected by Auger electron spectroscopy (AES). After about one hour of exposition in the residual vacuum, a trace of CO appeared in AES spectra. Flash heating to $850^{\circ} \mathrm{C}$ restored the clean surface. Sulphuration was performed by exposing the crystal to $5 \times 10^{-7}$ torr of $\mathrm{H}_{2} \mathrm{~S}$ at room temperature. Surface saturation was indicated by the ratio of $\mathrm{S} 152 \mathrm{eV}$ and $\mathrm{Pt} 237 \mathrm{eV}$ AES peaks. No correction was applied to the presented spectra for the variation of the analyser resolution and transmission or for inelastic electrons.

Figure 1 shows the most significant energy distribution curves (EDC's) of photoelectrons emitted normally to the clean surface. The same product total photocurrent $X$ scan time per energy

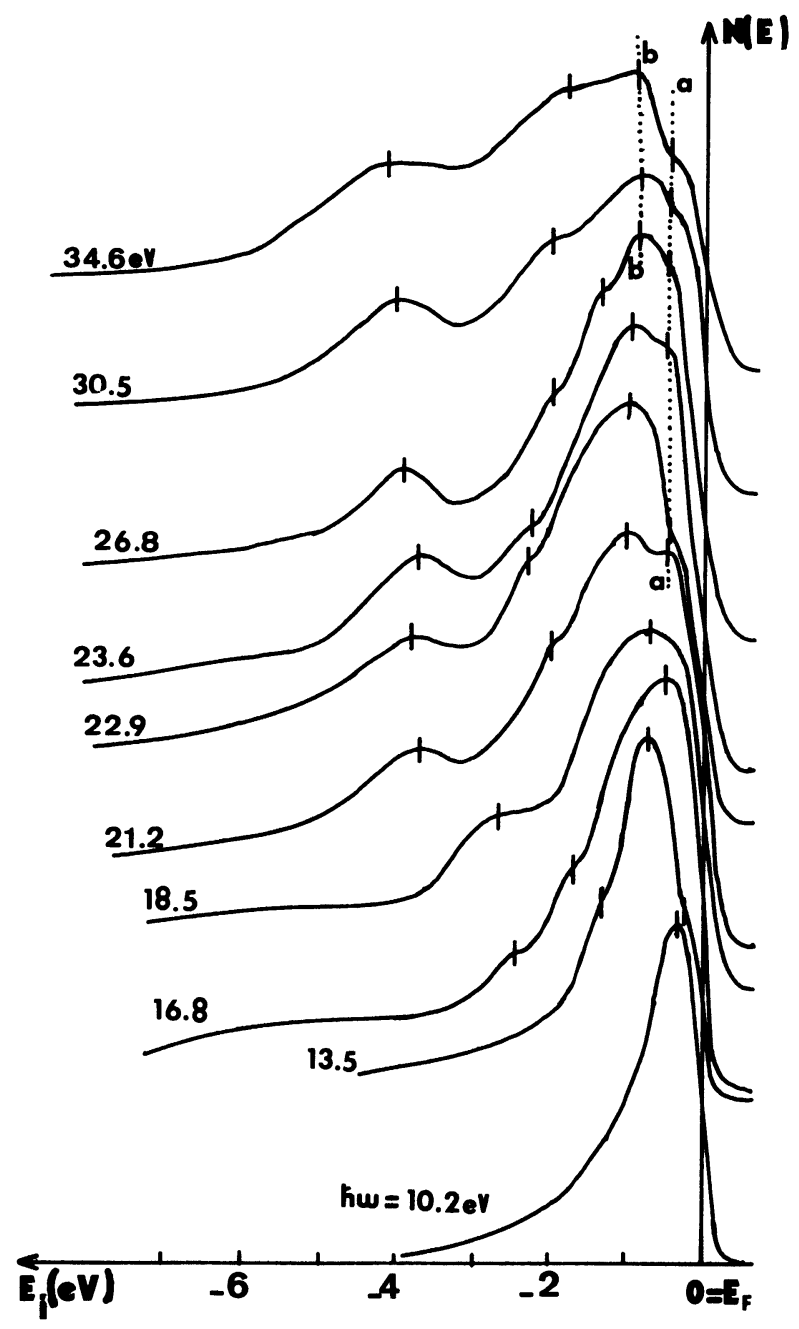

Fig. 1. - EDC's of photoelectrons emitted normally to the clean Pt (100) surface. Energy positions of the electronic structures are indicated by vertical bars; « $a$ " and « $b$ " : structures not originating from direct interband transitions (see text). 
unit was kept for all spectra. For low photon energies $(h v \leqslant 18.5 \mathrm{eV})$ the EDC's are composed of a dominant peak centred at about $0.5 \mathrm{eV}$ below the Fermi level and of weak shoulders. At higher $h v$, a second peak appears around $-4 \mathrm{eV}$. The rapid intensity decrease at the high binding energy side is probably due to the cut-off function of our analyser system.

Let us examine if the electronic structures, peaks or shoulders, appearing in these spectra originate from the three-dimensional density of states by direct interband transitions as generally observed in ARUPS. The valence band structure of platinum has been calculated by several authors : Anderson [9] by the RAPW method, Smith [4] using a combined interpolation scheme and more recently McDonald et al. [5] by a density functional formalism. Only Smith has given conduction bands up to $14 \mathrm{eV}$ above the Fermi level. These empty final states have a quasi-free electron character and can be drawn empirically if critical points are deduced from the EDC's, as we did before for $\mathrm{Pt}$ (111) [1]. This is not the case here for $\mathrm{Pt}(100)$ and for the following interpretation we use Smith's conduction band [4]. In figure 2, the experimental results are compared with the band structure along the $\Gamma \mathrm{X}$ direction of the $k$ space calculated for platinum by McDonald. Full circles indicate the energy positions of the experimental electronic structures, the peaks or shoulders of the EDC's in figure 1. The dashed lines correspond to the parabolic empty band of Smith shifted downwards by the corresponding photon energies. Each intersection point of the empty and filled bands corresponds to a possible direct interband transition. We see that nearly all the features of the spectra can be related to the three-dimensional density of states via direct interband transitions. The agreement between the experiments and the calculations of McDonald is fairly good, with deviations of less than $0.3 \mathrm{eV}$. The remaining structures which do not correspond to the three-dimension density of states belong to two stationary groups centred at about $-0.5 \mathrm{eV}$ and $-1 \mathrm{eV}$ below $E_{\mathrm{F}}$ and specified respectively by « $\mathrm{a}$ » and « $\mathrm{b}$ » on the EDC's of figure 1. Structure « $\mathbf{a}$ » is angle dependent, having its maximum at a collection angle equal to $30^{\circ}$ and as it does not disappear upon adsorption (Fig. 3) an eventual surface state origin is ruled out. A Fermi edge would be detected in similar experimental conditions at an energy closer to $E_{\mathrm{F}}$, less than $0.2 \mathrm{eV}$. For these reasons, we attribute it to a surface emission process from the high

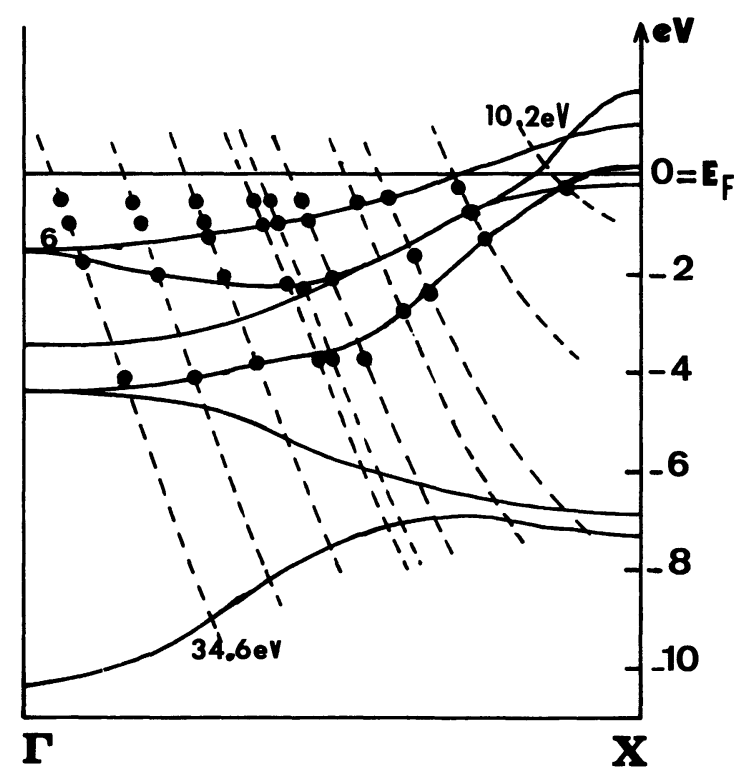

Fig. 2. - Band structure for Pt along the $\Gamma \mathrm{X}$ direction of the Brillouin zone. Full lines : dispersion curves calculated by McDonald [5] ; dashed lines : Smith's conduction band [4] shifted downwards by respective $\hbar \omega, 10.2,13.5 \ldots 34.6 \mathrm{eV}$; full circles : experimental results. 


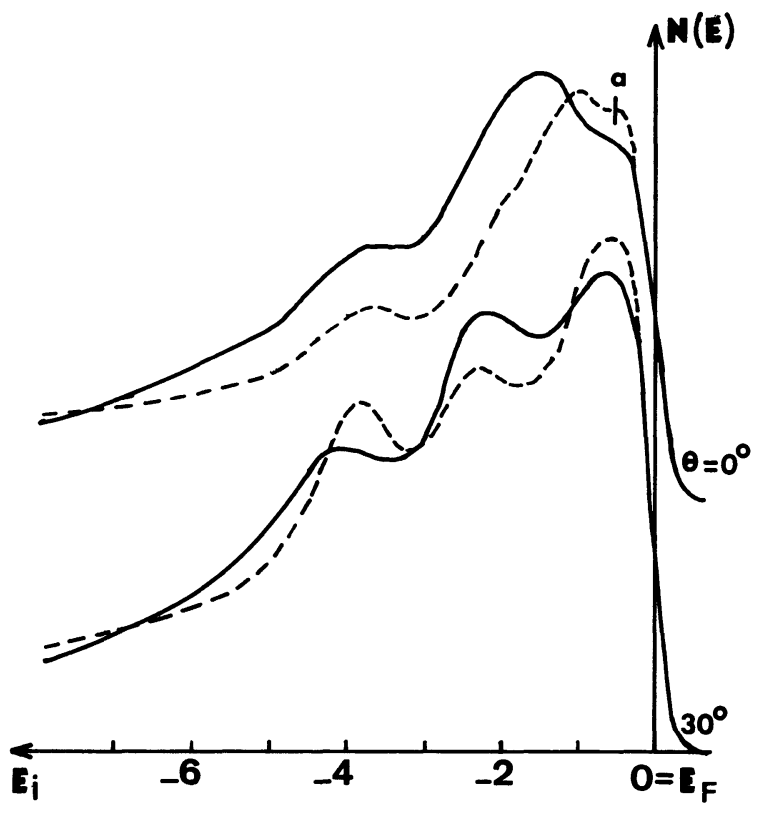

Fig. 3. - Variation of structure « $\mathrm{a}$ " with collection angle $\theta$ and with sulphur adsorption at $\hbar \omega=21.2 \mathrm{eV}$. Full lines : $\mathrm{S}$ saturated surface; dashed lines : clean surface.

density of states region near $E_{\mathrm{F}}$ observed with many transition metals, in a similar way as with $\mathrm{Pt}$ (111) [1]. The peak labelled « $\mathrm{b}$ » can possibly be related to the $(5 \times 20)$ reconstruction of the $\mathrm{Pt}$ (100) clean surface prepared by ion bombardment and annealing. The main argument supporting this hypothesis is that this structure decreases considerably upon sulphur adsorption as indicated by the $40.8 \mathrm{eV}$ and $26.8 \mathrm{eV}$ curves in figure 4 . It may also be favourably compared with the angle integrated UPS results of Helms et al. [10] who observed a difference in the spectra given by the classical reconstructed $(5 \times 20)$ surface and the unreconstructed one specially prepared by reaction of adsorbed $\mathrm{CO}$ with oxygen ions : the latter presented a sharp peak at $-0.25 \mathrm{eV}$ while the former had a softer one in the same energy region as our structure « $b$ ».

The only other ARUPS study of Pt (100) surface is that of Thornton et al. [3]. Using synchrotron radiation these authors have measured the normal EDC's in the photon energy range from 6 to $32 \mathrm{eV}$ and have drawn empirical dispersion relations. The final states used were deduced from a fitted $\mathrm{Au}$ conduction band [2], modified in order to take into account the differences in lattice constants and in $E_{\mathrm{F}}$ for the two metals. Agreement with the Anderson RAPW valence band [9] was qualitative, and except for the band 6, discrepancies were observed both in the energy positions and in the shape of the bands. As for supplementary structures, our interpretation is different from that of Thornton : they assigned the peaks near $-4 \mathrm{eV}$ to an intensity resonance of the one-dimensional density of states and the bulk density of states, while in our case these features follow interband transitions. We also note that Thornton et al. have not observed the structure we attribute to the reconstructed surface.

The EDC's of photoelectrons emitted normally to a surface saturated with adsorbed sulphur are reported in figure 4. Spectra of the clean surface are also shown by dashed lines, for comparison. Three main observations are given by these curves :

a) A broad but well defined extra electronic structure " $\mathrm{c}$ ", centred at about $6.5 \mathrm{eV}$ below $E_{\mathrm{F}}$, is visible in all the spectra taken at $\hbar \omega \geqslant 26.8 \mathrm{eV}$. 


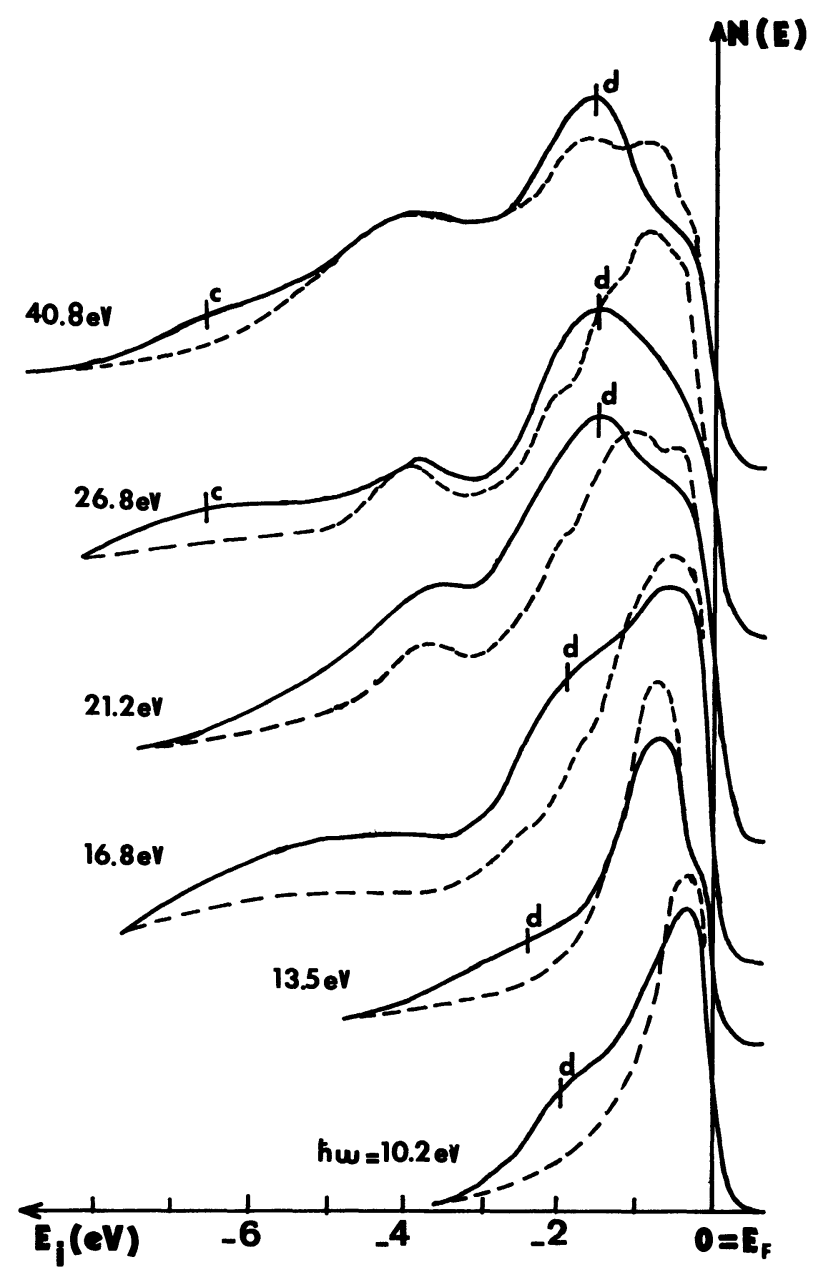

Fig. 4. - Normal emission spectra for sulphur adsorption. Full lines : S saturated surface; dashed lines : clean surface.

b) A second well defined structure « $\mathrm{d}$ » appears on all the curves, at around $2 \mathrm{eV}$ below $E_{\mathrm{F}}$. Its energy position moves slightly with $\hbar \omega$.

c) Except for the structure attributed to the $(5 \times 20)$ surface, the other metal electronic features are weakly attenuated by sulphur adsorption. On the contrary, for $\hbar \omega$ in the $16.8 \mathrm{eV}-21.2 \mathrm{eV}$ range, an important additional emission emerges between $-2 \mathrm{eV}$ and $-6.5 \mathrm{eV}$. Some of the metal structure are also slightly shifted.

This additional emission and these energy shifts restrict the use of classical difference curves for interpreting adsorption results. The two regular extra structures introduced by sulphur adsorption (peaks « $\mathrm{c} »$ and $" d »)$ are very similar to the ones detected on $\mathrm{Ni} \mathrm{(100)}$ surfaces [7]. This fact indicates that there is no fundamental difference in the chemisorption bonding of chalcogen with $5 \mathrm{~d}$ and $3 \mathrm{~d}$ transition metals, as has been suggested [6]. The deeper feature $(\sim-6.5 \mathrm{eV})$ can be readily attributed to the $3 p$ bonding states of sulphur because of the correspondence with the ionization energy of $S 3 p$ orbitals and the analogy with previous studies of chalcogen adsorption on transition metals $[7,11,12,13]$. As for the shallower level $(\sim-2 \mathrm{eV})$, its origin cannot be 
straightforwardly determined. Its similarity with the -1.8 peak in ARUPS study of $S$ and $O$ adsorbed on $\mathrm{Ni}(100)$ [8] indicates that it would be due to the $\mathrm{d}$ metal states localized by the presence of adsorbate. This is perhaps the most interesting part of our adsorption results : the evidence of the presence of perturbed metal states which is rarely taken into account in the studies of the electronic character of adsorption.

\section{References}

[1] Cinti, R., Nguyen, T. T. A. and Capiomont, Y., J. Physique-Lett. 40 (1979) L-377.

[2] Mills, K., Davis, R. F., Kevan, S. D., Thornton, G. and Shirley, D. A., Phys. Rev. B 22 (1980) 581.

[3] Thornton, G., Davis, R. F., Mills, K. A. and Shirley, D. A., Solid State Commun. 34 (1980) 87.

[4] Smith, N. V., Phys. Rev. B9 (1974) 1365.

[5] McDonald, A. H., Daams, J. D., Vosko, S. H. and Koelling, D. D., Phys. Rev. B23 (1981) 6377.

[6] Collins, D. M. and SPICER, W. E., Surf. Sci. 69 (1977) 114.

[7] KüPPERS, J. and ERTL, G., Surf. Sci. 77 (1978) L647.

[8] Nguyen, T. T. A., Cinti, R. and Capiomont, Y., Surf. Sci. 87 (1979) 613.

[9] Andersen, O. K., Phys. Rev. B 2 (1970) 863.

[10] Helms, C. R., Bonzel, H. P. and Keleman, S., J. Chem. Phys. 65 (1976) 1773.

[11] Fischer, T. E. and Kelemen, S. R., Surf. Sci. 69 (1977) 1.

[12] Hagstrum, H. D. and Becker, G. E., J. Vacuum Sci. Technol. 14 (1977) 369.

[13] JaCobi, K., Scheffler, M., Kambe, K. and Forstmann, F., Solid State Commun. 22 (1977) 17. 